\title{
Antropología e historia: Notas para una etnografía del pasado
}

\author{
Anthropology and history: \\ Notes for an ethnography of the past
}

William Andrés Martínez-Dueñas ${ }^{1} \&$ Astrid Lorena Perafán-Ledezma ${ }^{2}$

\section{Resumen}

Este documento trata sobre la relación entre la antropología y la historia, y sobre cómo abordar el pasado en el proceso etnográfico. Para esto trabajaremos con un grupo de autores quienes, desde perspectivas etnográficas, han abordado la historia y/o el pasado. Veremos esto aplicado a procesos político-organizativos, el análisis etnográfico de la lucha por tierras y la re-interpretación de los eventos del pasado desde sus diferentes agentes y situaciones. Esto hará evidente cómo los relatos históricos son de diferente naturaleza y obedecen a diversos objetivos, desde visibilizar las relaciones de poder dentro del relato, hasta los fines prácticos que justifican la narración del pasado y cómo la etnografía es una herramienta útil para abordar estos procesos.

Palabras clave: Etnografía; historia; etnohistoria; pasado

\begin{abstract}
This article centers around the relationship between anthropology and history, specifically around how the past is dealt with in ethnographic processes. Several authors are reviewed, who have approached history and/or the past from ethnographic perspectives. Such approaches are then studied in organizational political processes, in ethnographic accounts of fights for land, and in the re-interpretation of past events from the perspective of different actors and agents. By this means, it will be clear that historical narratives can be told from different angles and can have different goals, from rendering power relationships evident to attempting to achieve practical wins. Ethnography is, thus, a useful tool for approaching such processes.
\end{abstract}

Keywords: Ethnography, history; ethnohistory; past

Tipología: Artículo de reflexión

Recibido: $28 / 11 / 2016$

Evaluado: 05/01/2017

Aceptado: 19/01/2017

Disponible en línea: 01/03/2017

Como citar este artículo: Martínez-Dueñas, W.A. \& Perafán-Ledezma, A.L. (2017). Antropología e historia: notas para una etnografía del pasado. Jangwa Pana, 16 (1), 67 - 75. Doi: http://dx.doi.org/10.21676/16574923.1957

1 .Dr. Antropología. Profesor asociado programa de Antropología, Facultad de Humanidades, Universidad del Magdalena. Colombia. Correo electrónico:wmartinez@unimagdalena.edu.co. ORCID ID: 0000-0003-0921-1149

2. Dr. Antropología. Profesora asociada programa de antropología, Facultad de Humanidades, Universidad del Magdalena. Colombia. Correo electrónico: aperafan@unimagdalena.edu.co. ORCID ID: 0000-0003-4377-7170 


\section{Introducción}

La historia etnográfica o la etnografía del pasado, puede entenderse como la apuesta teóricametodológica que permite abordar cómo el pasado (lo que paso y lo que se dice que pasó) es construido y resignificado en el presente, a través de la relación que los sujetos y las comunidades establecen con los archivos, las prácticas socio-culturales, los lugares, los paisajes y las historias orales. La historia etnográfica se centra en describir y analizar el uso de la relación entre la fuente escrita, la oral y el territorio en los procesos politico-organizativos, pedagógicos, de construcción y resignificación de identidades. Además, busca abordar las prácticas de producción histórica en lo local o de construcción del pasado en lo cotidiano, articulado con procesos culturales, históricos, sociales y políticos más amplios. Es importante aclarar que se entiende lo etnográfico como un método de producción de conocimiento con los otros y no sobre el Otro, donde se evita la negación de la coetaniedad, es decir la suposición que el Otro vive en otro tiempo (Fabian, 1983) y el etnocentrismo (Derrida, 2000), además de reducir la relevancia de la positividad en el relato histórico. Esto marca una diferencia con la etnohistoria ${ }^{1}$, donde el objetivo de usar los métodos etnográficos es la acumulación de información empírica "verificable" que incremente el valor de verdad o positividad de la historia (Welters, 1992).

Este documento tiene como objetivo explorar la relación entre la antropología y la historia, enfocándose en lo que hemos denominado etnografía del pasado. Retomaremos un grupo de autores quienes han abordado la historia y/o el pasado a través de análisis etnográficos sobre la re-interpretación de los eventos del pasado desde sus diferentes actores y situaciones (Trouillot, 1995; Amin, 1995), el análisis etnográfico de la lucha por tierras (Rappaport, 2005) y los procesos político-organizativos (CRIC, 2004; Fals Borda, 1979). Esta exploración nos permitirá evidenciar que los relatos históricos son de diferente naturaleza, que obedecen a diversos objetivos e intereses, y la manera en que se construyen en función de los loci de enunciación de los investigadores y actores involucrados en la construcción del relato. Este análisis nos llevará desde la construcción del pasado en el presente hasta los usos prácticos del relato histórico.

\section{Antropología del encuentro-creación con el pasado}

La historia etnográfica se puede entender como un enfoque teórico-metodológico que indaga cómo en el presente se construye el pasado, donde los diferentes actores construyen múltiples narrativas sobre el pasado, dando uso e interpretando de forma distinta los documentos históricos, los archivos y los documentos judiciales en relación con las narrativas e historias locales. Los actores privilegian y construyen ciertos eventos, archivos y narraciones locales acordes a las circunstancias y particularidades en las cuales se encuentran inmersos. Desde este punto de vista, el interés en la producción de los relatos históricos, no tiene pretensiones de esclarecer la verdad, sino que se centra en analizar el sentido pragmático y heterogéneo (Amin, 1995) de la elaboración de los mismos. Esto es posible debelarlo a través de la observación de la creación misma de la historia y del análisis crítico de las historias oficiales contrastándolas con las no oficiales (subalternas, locales, las stories) (Amin, 1995; Trouillot, 1995; Rappaport, 2005).

El encuentro-creación con el pasado se da de múltiples maneras; sea a partir de los relatos orales, de los documentos legales y no legales, de la cultura material, los rituales y el territorio. Desde esta óptica la historia etnográfica no se centra exclusivamente en lo oral, como acostumbran los antropólogos, o en lo escrito, como acostumbran los historiadores, sino que por el contrario se centra en analizar la producción histórica como "un palimpsesto en donde lo oral y lo escrito coinciden en parte, se mezclan y se reinterpretan una y otra vez con nuevos actores" (Rappaport, 2005, p.188).

1. También puede entenderse etnohistoria como la historia de las culturas y las sociedades o el estudio del pasado de los otros (Pannabecker, 1990). 
Por ejemplo Rappaport (2005), con el fin de establecer el proceso de producción de la historia en los renacientes de Cumbe (Nariño - Colombia), utilizó dos estrategias generales que le permitieron construir un modelo multiestratificado, polifónico y dialógico de la creación del presente como pasado. Una de las estrategias es la observación participante a partir de la cual se pudo establecer cómo en la vida cotidiana, en los rituales, en la transmisión de relatos orales, la significación de la cultura material y el análisis de documentos legales y personales se recrea constantemente la historia como un performance continuo atravesado por relaciones de poder tanto internas como externas al grupo que lo produce. La segunda estrategia es el trabajo de archivo no como una actividad aislada que busca verificar los relatos históricos, sino que evidencia la manera en que la documentación pública y privada es interpretada y re-interpretada en la producción de dichos relatos históricos. Esto permite no sólo definir la producción cultural como estrictamente oral sino también establecer cómo se da la relación entre discursos (orales y escritos) hegemónicos y subalternos. De esta forma se llega a una etnografía del pasado que vincula en un mismo análisis lo oral y lo escrito, junto con la cultura material y las expresiones rituales. Así la producción de la historia se entiende en un devenir constante, y la tarea del antropólogo, es la de describir el proceso y los aspectos particulares que en un momento específico determinan la producción de los relatos históricos.

El investigador no usa la información de archivo como evidencia probatoria o rectificadora de la historia relatada por los actores locales, sino que analiza "la yuxtaposición de una amplia gama de información en procura de comprender cómo una comunidad indígena que vive bajo coerciones sociales y condiciones históricas específicas, aprende a interpretar su propio pasado" (Rappaport, 2005, p. 43). La red que reconstruye el antropólogo incluye la narrativa oral y los rituales, los rasgos del paisaje, afiches publicitarios, documentos legales, archivos comunales, entre otros. Así se logra, como plantea Rappaport, "tratar el material histórico como fuente para los intelectuales locales, en vez de usarlo como evidencia para mi reconstrucción histórica” (Rappaport, 2005, p. 43).

La sobre-posición de lo oral y lo escrito en la producción de la historia evidencia también como ésta es relevante para el presente, es decir, evidencia el valor del pasado en el presente inmerso en una serie de relaciones de poder. En Cumbe renaciente se puede observar cómo en la cotidianidad se produce la sobre-posición o el palimpsesto de la producción histórica; de esta manera el antropólogo no puede analizar documentos por un lado y describir prácticas y compilar relatos orales por otro, por el contrario, la propuesta metodológica de la etnografía del pasado ve la interacción entre lo escrito y lo oral en la cotidianidad donde el pasado es presente.

Al hablar del pasado como presente está implícita la performatividad histórica, ya que la representación del pasado no es exclusiva de ciertos rituales o expresiones artísticas locales, sino que el territorio en toda su extensión es un gran escenario donde se recrea constantemente la historia, involucrando a toda la comunidad, los rasgos topográficos del territorio y la cultura material sobre este (Rappaport, 2005). Y en un sentido todavía más general, el pasado como presente es un pasado que deviene diferente constantemente, actualizándose a través de las prácticas cotidianas y las luchas políticas y económicas, donde el etnógrafo de la historia presencia la producción misma del pasado e inclusive interviene en su producción.

De una manera similar Trouillot (1995), aunque no utiliza el término etnografía como sí lo hace Rappaport, estudia la multiplicidad de relatos que se producen frente a un hecho histórico y cómo en cada relato se incluyen una serie de silencios. Trouillot sugiere analizar la producción histórica a través de la yuxtaposición de los hechos socio-históricos con las narrativas históricas, lo cual se diferencia de las apuestas positivistas y constructivistas sobre la historia, donde la primera intenta teorizar la distinción entre el proceso histórico y la narrativa, garantizando la positividad de la historia; y la segunda, por su parte entiende la narrativa histórica como una ficción 
más (Trouillot, 1995, p. 165). En términos generales Trouillot menciona dos momentos principales en la producción del pasado. El primer momento del que habla es uno material, donde la historia comienza con cuerpos y artefactos: cerebros vivientes, fósiles, textos, entre otros; es decir una colectividad de humanos y objetos en interacción, produciendo acontecimientos (historicidad 1). Esa producción de acontecimientos establece el escenario de futuras narrativas históricas que se constituye como el segundo gran momento (historicidad 2). Estos dos momentos generales pueden ser desglosados en una serie, progresiva pero diversa, de silenciamientos en la producción de los relatos históricos (Trouillot, 1995, p. 178):

a) el momento de la creación del hecho (la construcción de las fuentes)

b) el momento de la recolección de los hechos (construcción de archivos)

c) el momento de la recuperación de los hechos (construcción de narrativas)

d) el momento de la significación retrospectiva (la construcción de la historia en última instancia).

En cada uno de los momentos anteriores hay un proceso de silenciamiento en el que los sujetos, conscientes o inconscientemente, producen conocimiento sobre "lo que pasó" haciendo descripciones parciales; algunas partes del hecho no se dicen y otras por el contrario son dichas con mayor énfasis. Así mismo, en la construcción de archivos y de narrativas, hay silenciamientos y énfasis que influyen sustancialmente en la síntesis histórica última. Trouillot llama la atención en la multiplicidad de silencios que se desprenden de un mismo hecho histórico, y sugiere no solo fijarse en uno sino más bien ver cómo están relacionados con sus contextos y con los otros relatos e historias. Esto último lleva a que la historia sea entendida como una producción de silencios y menciones que, al analizarse de manera transversal, pone en evidencia el carácter polifónico de la historia.

Un ejemplo de lo anterior, se puede hacer evidente en el análisis que plantea Alexander $(2003)^{2}$, quien

2. Vale la pena aclarar que Alexander no retoma el trabajo de Trouillot, pero creemos que el ejemplo a ser ampliamente conocido, puede ser esclarecedor. hace visible cómo la historia del holocausto judío da cuenta de una narrativa construida desde aquellos que tenían el control sobre los medios de producción simbólica: la sociedad estadounidense, silenciando las versiones de los otros actores centrales del evento. En esta narrativa los protagonistas no eran los judíos, sino los soldados estadounidenses, quienes aparecían como los sujetos liberadores, moralmente justos y buenos, y los nazis como la representación del mal absoluto. Estas narrativas serian diferentes si quien las narrara fuera un soldado, un oficial, un periodista o un historiador. Así mismo habría variantes si quien hablara sobre los hechos fuera judío, estadounidense o alemán. Cada quien habla o calla según sus intereses, enfatizando o silenciando según su conveniencia.

Para Trouillot la historia es un proceso de sobreposición entre el hecho histórico (lo que pasó) y lo que se dice del hecho histórico, donde los narradores son actores y viceversa. Esto es un punto de encuentro con Rappaport, salvo que para ella el énfasis es la producción constante en lo cotidiano del relato histórico, mientras que Trouillot implementa un mayor trabajo sobre lo que se dice del hecho en sus diferentes versiones y contextos. El trabajo de Trouillot es etnográfico en la medida en que este escucha la multiplicidad de relatos históricos y cómo estos producen silencios diferentes; estos relatos no sólo son producidos por historiadores profesionales sino que son elaborados, debatidos y clasificados por líderes étnicos y religiosos, políticos, periodistas, organizaciones civiles $\mathrm{y}$ ciudadanos independientes. Es precisamente ésta multiplicidad de sujetos a quienes la historia ha opacado y restado importancia. En esta medida los eventos y sus narrativas cambian, se validan o invalidan de acuerdo a los actores, su lugar de enunciación, la relación o no con el hecho y lo que se dice de este. Por lo tanto, un enfoque etnográfico como el que implementa Trouillot, permite evidenciar la polifonía silenciada en los relatos históricos en general.

Tanto Trouillot como Rappaport se interesan en la producción de la historia, en cómo se produce conocimiento histórico y cómo ésta producción está inmersa en relaciones de poder (construcción 
de identidad, organización indígena, tenencia de la tierra, acceso a la toma de decisiones) que determinan las narraciones históricas y que dinamizan su devenir constante. En términos generales estos dos autores estudian la relación entre lo que pasó y lo que se dice sobre lo que pasó, la diferencia radica en el énfasis que hace Rappaport en la observación participante y en cómo se re-produce la historia en la cotidianidad. Mientras que Trouillot analiza los procesos de silenciamiento en las diferentes versiones que describen y significan un hecho histórico como por ejemplo el del Álamo33. Rappaport experimenta, al calor de un fogón, cómo se actualiza el relato sobre Cumbe y la lucha por la tierra. Trouillot lee los diferentes relatos y analiza los silencios que los caracterizan según el contexto en el que fueron producidos, mientras que Rappaport presencia el performance histórico en tiempo real.

El enfoque de Trouillot es etnográfico porque analiza cómo se da el proceso de construcción histórica, de la misma manera como los antropólogos estudian o analizan el proceso de producción y transformación de la cultura. A pesar de que Trouillot no presencia los hechos que serán tornados en historia y la producción de las narraciones con relación a dichos hechos, si establece cómo la producción de dichos relatos está determinada por su contexto y como ya se mencionó, Trouillot describe la polifonía silenciada en los diferentes relatos históricos al no sólo estudiar lo que dicen los historiadores profesionales, sino lo que los otros actores y sujetos de la historia dicen sobre lo que pasó, así como eso que se dice que pasó influye en el presente.

Con un sentido similar Amin muestra la imposibilidad de la síntesis histórica y permite ver el pasado como multiplicidad y devenir. Amin (1995) plantea la imposibilidad de construir una historia general sobre los acontecimientos dados, refiriéndose en este caso

\footnotetext{
3. Fuerte apodado el Álamo, ubicado en la misión de San Antonio de Valero en la provincia de Tejas (México) donde, en marzo de 1836, el ejército mexicano comandado por el general Antonio López de Santa Anna, después de 12 días de batalla, tomó el fuerte el 6 de marzo. (Trouillot, 1995). De acuerdo con Trouillot (1995), sobre esta batalla se ha configurado una serie de controversias y debates que cuestionan si la batalla es una expresión de libertad, heroísmo y sacrifico de los norteamericanos o representa el expansionismo norteamericano.
}

a Chauri Chaura (India) ${ }^{4}$ en 1922, ya que los eventos, la construcción de las fuentes, el archivo y la historia misma son diversas y divergentes, así como sus usos de acuerdo al lugar, el tiempo y al locus de enunciación de los actores, ya sea como testigos, parientes de los policías quemados, parientes de los generadores de la revuelta, parientes tanto de policías como de los generadores de la revuelta y vecinos del lugar. Al respecto Amin (1995) nos muestra cómo la revuelta de Chauri Chaura, inicialmente fue repudiada y excluida del calendario nacionalista por ir en contra de la ideología de la no violencia, pero posteriormente con la independencia de la India, la protesta fue considera como heroica e incluida dentro de la historia oficial de la Nación (Amin, 1995).

\section{Historia para}

La historia etnográfica propone considerar otras formas de construir el pasado más allá de los relatos lineales, evidenciando su relación con el presente, su carácter dinámico, heterogéneo y móvil. El pasado se construye colectivamente en el presente, "...el pasado no existe independiente del presente; de hecho, el pasado sólo es pasado porque hay un presente, de la misma manera que puedo señalar algo que está allá porque yo estoy aqui" (Trouillot, 2007, p. 171). Desde perspectivas no occidentales el pasado es entendido de maneras diferentes, y en tal medida la historia etnográfica es capaz de evidenciar estas diferencias. Por ejemplo para los guambianos (Cauca, Colombia) "el pasado está adelante, es merrap, lo que ya fue y va adelante; went $\varnothing$ es lo que va a ser, lo que viene atrás. Entonces lo que no ha sido viene caminando de atrás" (Vasco, Dagua \& Aranda, 1993, p. 11) ${ }^{5}$.

\footnotetext{
4. Revuelta protagonizada campesinos en abril de 1922 en Chauri Chaura, al norte de India, quienes motivados por la victoria de Mahatma Gandhi quemaron la estación de policía de la localidad y dieron muerte a un grupo de policías (Amin, 1995).

5. Es importante resaltar que para comunidades indígenas del Cauca (Colombia), como los Guambianos y los Nasa, se pueden presentar múltiples formas de narrar el pasado: en espiral, lineal, en relación con la topografía, entre otras, que están en función de las condiciones en que se construye el relato (Rappaport, comunicación personal) (ver también Rappaport, 2000). De manera similar Martínez-Dueñas (2016) mostró cómo en los relatos sobre el agua en el Resguardo Indígena de Puracé (Cauca- Colombia) se mezclan los tiempos y los seres modernos con los tiempos (el tiempo de los antiguos) y los seres no modernos (e.g. El Duende y El Espíritu del Agua), donde dichos relatos representan un mundo no[solo]moderno.
} 
En el caso de los cumbales (Nariño) la historia no es vista hacia atrás, el "pasado se localiza frente al observador, confiere un aura histórica a las actividades del presente" (Rappaport, 2005, p. 88) y no está organizado linealmente, sino de maneras heterogéneas (Rappaport, 2005, p. 87). Entonces,

[s]i el conocimiento histórico está ligado al presente a través de esta manifestación nolineal en el espacio y en el ritual se debe a que tiene aplicaciones prácticas. El conocimiento del pasado es un componente fundamental en las disputas por tierras, los acuerdos políticos y las discusiones sobre las herencias (Rappaport, 2000, p. 42).

Si bien Trouillot y Rappaport analizan los procesos de producción de la historia desde perspectivas que tienen en cuenta las relaciones de poder que atraviesan los relatos que producen la historia, su análisis es principalmente académico a pesar de la potencia política del mismo. Este tipo de análisis se diferencia de los llevados a cabo por el Consejo Regional Indígena del Cauca (CRIC, 2004) y Fals Borda (1979) en los que la construcción del relato histórico tiene un fin político directo, por ejemplo de colaboración e intervención académicopolítica (caso Fals Borda) o un posicionamiento intercultural (caso CRIC).

Fals Borda recurre a la etnografía para obtener información sobre el pasado que permita "adelantar la tarea de recuperar la historia de Loba [Colombia] y animar con ella la acción concreta que beneficie a los sufridos habitantes de la depresión momposina" (1979, p. 29A). Fals Borda empleó una técnica de investigación que denominó imputación

[...] que combina la información sobre los hechos con la reflexión y la comunicación basadas en observaciones sucesivas (...) hace declarar hechos u observaciones demostradas que desbordan sus declaraciones literarias, las mismas que puedan constar en diarios de campo o en cintas magnetofónicas. (Fals Borda, 1979, p. 26B-27B)

La información oral colectada es complementada con documentación oficial y de "baúl" o local no oficial, todo esto dentro del marco de la investigación acción donde se destacan dos procesos principales: a) la recuperaron crítica, que es una "técnica de examen e interpretación, con el enfoque de clases explotadas", que también podemos entender como producir relatos históricos de la explotación y la dominación y b) la devolución sistemática, que consiste en comunicar dicha historia de la explotación para educar y propiciar la acción política, lo que nos lleva a proponer este tipo de producción de relatos históricos como una historia para la acción. La orientación política del análisis se hace evidente en la siguiente afirmación de uno de los actores locales: "habrá que recuperar esa historia, admito entusiasmado, y devolverla y ponerla al servicio de los actuales intereses de los campesinos lobanos y de toda la región momposina" (Fals Borda, 1979, p. 29A).

Fals Borda, a pesar de incorporar a un grupo de habitantes de la región para reconstruir la historia de Loba y Mompox (Colombia) y de plasmar las voces de ellos en el denominado Canal $\mathrm{A}^{6}$, en otro canal, Canal B, interpreta desde un análisis teórico la información recolectada para plasmar desde el lenguaje académico y de forma "fidedigna" y "exacta" la historia de Loba y Mompox, para ligarla al presente y ponerla al servicio de las luchas sociales. Como apuesta política busca

[...] hallar el alma colectiva de la región y su mundo, de entender los fundamentos de su realidad y la razón de sus problemas, de propiciar una útil transformación en la vida de las gentes (...) para defender la cultura local, sus aspectos positivos e impedir los estragos del sistema dominante. (Fals Borda, 1979, p. 28A)

Desde el punto de vista de Fals Borda, así como de Trouillot (1995), puede decirse que tanto los informantes (como los denomina Fals Borda), como el investigador académico mismo son sujetos de la historia, es decir, son voces conscientes de su vocalidad e intencionalidad que definen los términos bajo los cuales pueden ser descritas ciertas situaciones. La producción histórica en este caso

6. El canal A hace referencia a las voces de los habitantes de Loba y Mompox, y en el canal $\mathrm{B}$ el autor analiza las dinámicas históricas a la luz de la teoría académica. 
resalta los procesos de explotación y dominación que permitan a las comunidades contemporáneas, pertenecientes a las clases populares, impulsar "luchas justas" contra sus enemigos tradicionales. Fals Borda, al igual que Trouillot y Amin, evidencia que la historia "oficial" es una historia hegemónica atravesada por silencios (silencios de clase diría Fals Borda), como lo evidenció Fals Borda en las guerras cimarronas o Trouillot en la revolución haitiana, por lo tanto, se hacen importantes las historias locales o alternativas que al igual que las de élite varían según la posición ideológica, etárea $y$ de género de quienes aportan en la producción del pasado.

Otro tipo de historia para está representada en la monografía histórica "¿Qué pasaría si la escuela...? 30 años de construcción de una educación propia" escrita por el colectivo indígena encargado del Programa de Educación Bilingüe e Intercultural (PEBI) del Consejo Regional Indígena del Cauca (CRIC). Este documento tiene como objetivo escribir la historia del programa de Educación Bilingüe con el fin de

[...] reflexionar sobre la dinámica del proceso, los esfuerzos que hemos hecho por generar conceptos, en vez de aceptarlos como dados o como estáticos. Es decir, reconstruir nuestra historia nos permite dar una mirada compleja a nuestra experiencia. Además, al emprender una investigación histórica era necesario recoger las voces desde el contexto de la lucha, colectivizando así el proceso investigativo y uniendo lo local con los procesos regionales y la dinámica general de la organización. (CRIC, 2004, p. 25-26).

A diferencia de Rappaport y Trouillot los referentes teórico-conceptuales de este texto no tratan sobre el proceso de la producción histórica, sino que desarrollan tres conceptos claves: comunitariedad, cosmovisión e interculturalidad, relacionados con la dinámica político-organizativa del CRIC. La historia del PEBI cabe perfectamente en lo que Nora (citado por Rappaport, 2005) denomina memoria, entendida esta como
[...] vida, llevada por los grupos vivientes, y en sus nombres se halla un flujo permanente, abierta a la dialéctica del recuerdo y el olvido, inconsciente de sus sucesivas deformaciones, vulnerable a todos sus usos y manipulaciones, susceptible a largos periodos de latencia y a repentinas revitalizaciones (...) la memoria es un fenómeno todavía activo, un lazo viviente con el presente eterno (...) la memoria está arraigada en lo concreto, en el espacio, gestos, imagen y objeto. (Rappaport, 2005, p. 235-236)

Se puede ver que para el CRIC la historia del PEBI debe evidenciar cómo "las escuelas bilingües deben ser semilleros para revitalizar las culturas" (CRIC, 2004, p. 31); así mismo, mostrar el proceso de producción de la cosmovisión (más allá de costumbre y de la cultura), entendida como " $\mathrm{el}$ proceso de creación de dispositivos para analizar el mundo y actuar en el" lo cual está inmerso en "el proceso político-organizativo y en el contexto de una educación propia" (CRIC, 2004, p. 89); así mismo "el movimiento indígena retoma la tradición pero agrega algo que hoy necesitamos para responder a la realidad de hoy" (CRIC, 2004, p. 156). A partir de lo anterior y retomando la distinción que hace Nora entre historia, como la reconstrucción de lo que ya no es y memoria como vida, se puede afirmar que el documento del CRIC es más que historia, no es ausencia, sino vida, intervención de la realidad, creación que revitaliza y aviva los procesos políticos y comunitarios sin distinguir entre lo teórico y lo práctico; como diría Foucault y Deleuze, (1999 [1972]: 107) es "un sistema regional de lucha (...) [y] una caja de herramientas" para la construcción de comunidad-nación.

\section{Consideraciones finales: Ios loci de enunciación}

Estas otras formas de definir la historia y el pasado, claramente se asocian con el locus de enunciación y las apuestas políticas, culturales y cosmológicas de sus sujetos, lo cual nos permite hacer evidente las limitaciones de la manera cartesiana, lineal y positivista de abordar el pasado. Los ejemplos 
citados anteriormente (Trouillot, Rappaport, Amin, Fals Borda y el CRIC) nos llevan a reflexionar sobre cómo la historia, al igual que la antropología, aborda los procesos y las dinámicas culturales en el tiempo y en el espacio; nos invitan a pensar en los procesos y las prácticas culturales contemporáneas locales específicas, conectados con procesos de más larga duración y a verlos de forma dinámica, en constante comunicación y transformación. Así mismo, y como diría de Certeau, propone abordar el uso del pasado y no el pasado como tal, para lo cual se hace fundamental la reconstrucción de la trayectoria de los puntos recorridos, la evocación del recorrido diacrónico, el movimiento, el desplazamiento y no solo la descripción de unos puntos en un lugar sincrónico o acrónico (de Certeau, 2000, p. 42). En esta medida la etnografía comporta una dimensión histórica y evita la perspectiva estática sobre el Otro.

Todos los autores abordados en este documento, que también son sujetos de la historia, parten del encuentro con las historias no oficiales, las historias alternativas, historias no-modernas y las historias subalternas, donde la vía de exploración inicial es el conjunto de técnicas, de formas de escribir y de pensar que se denominan etnografía, así como la búsqueda de nuevos archivos que han escapado de las historias oficiales y que pueden constituirse en el combustible para diversos procesos político-sociales. Estas perspectivas presentan un posicionamiento político o unos loci de enunciación específicos, no solo desde lo académico, sino también desde lo político y geográfico, en donde podemos visibilizar posiciones solidarias con las causas políticas locales, combinadas en algunos casos con un pensamiento intercultural.

En el caso de Rappaport, aunque su texto es de corte académico, ella era consciente del potencial político de éste, lo cual direccionó parte de la investigación; la condición de la autora de ser solidaria con las luchas indígenas por la tierra no sólo aportó nueva información histórica, sino que también contribuyó en la dinámica político-organizativa del encuentro/ creación con el pasado. Así mismo puede especularse que el origen haitiano de Trouillot haya determinado sustancialmente su interpretación del proceso de producción histórica, ya que no se encuentra en una posición totalmente externa, como la que asumiría un investigador extranjero, sino que por el contrario la forma de entender el proceso histórico de su propio país es seguramente mucho más cercana y menos aséptica. En su caso habría de considerarse además la condición de ser un académico haitiano radicado en Norteamérica, lo cual lo sitúa en una suerte de frontera que privilegia aún más un análisis no etnocéntrico. Esto último es quizá lo más importante, más que el hecho de que Trouillot sea haitiano, es el hecho de ser un pensador de frontera, tanto social como académicamente, lo que hace que sus análisis sean más ricos y aborden la historia de una manera crítica y diferente (quizá comparable con el trabajo de Edward Said y los del PEBI, mencionado arriba).

En el caso de Fals Borda, al ser un militante marxista, es muy claro el objetivo político de su trabajo de investigación acción participante, o IAP, dentro de procesos políticos concretos, lo cual es evidente cuando afirma que él es un "observador barranquillero de origen momposino que no quería volver a escribir libros de sociología, a menos que resolvieran la cuadratura del círculo de la comunicación social con organizaciones de diversos intereses de clase y nivel intelectual" (Fals Borda, 1979, p. 17A [nótese que está en el canal A]).

Por su parte la historia del PEBI del CRIC a la cabeza de dos "intelectuales indígenas", Graciela Bolaños y Abelardo Ramos, y una "investigadora universitaria solidaria" con la organización desde hace décadas, Joanne Rappaport, en co-autoría con otros actores de la organización indígena, presenta un locus de enunciación intercultural y una doble conciencia como ellos mismos lo afirman, dando origen a un pluri-locus fronterizo, que revindica la historia local como poder en el presente para las luchas de la organización-comunidad. 


\section{Referencias bibliográficas}

Alexander, J. (2003). The Meanings of Social Life. A Cultural Sociology, Oxford: Oxford University Press.

Amin, S. (1995). Event, Metaphor, Memory: Chauri Chaura 1922-1992. Berkeley: University of California Press.

CRIC. (2004). ¿Qué pasaría si la escuela. . .? Treinta años de construcción educativa. Popayán: Programa de Educación Bilingüe e Intercultural, Consejo Regional Indígena del Cauca.

De Certeau, M. (2000). La invención de lo cotidiano I. Artes de hacer. Universidad Iberoamericana. México: Departamento de Historia Instituto tecnológico y de estudios superiores de occidente.

Derrida, Jacques. (2000). De la gramatología. México: Siglo XXI.

Fabian, J. (1983). Time and the other. Nueva York: Columbia University Press.

Fals Borda, O. (1979) Mompox y Loba: Historia doble de la Costa. Bogotá: Carlos Valencia Editores.

Foucault, M. \& Deleuze, G. (1999). Los intelectuales y el poder. En: Estrategias de Poder. Obras esenciales, Volumen II (pp. 105-115). Barcelona: Paidós

Martínez-Dueñas, W. (2016). Flujos y redes multinaturales: un recorrido por mundos no[solo]modernos en Puracé, Colombia. Popayán: Editorial Universidad del Cauca.

Pannabecker, R. (1990). Linking Anthropology and History in Textiles and Clothing Research: The Ethnohistorical Method Clothing and Textiles Research Journal, 8 (3), 14-18.

Rappaport, J. (2005), Cumbe renaciente: Una historia etnográfica andina Bogotá: ICANH/Popayán: Editorial Universidad del Cauca.

Rappaport, J. (2000). La política de la memoria: interpretación indígena de la historia en los andes colombianos. Traducido por Jóse Ramón Jouvé. Popayán: Editorial Universidad del Cauca.

Trouillot, M.R. (1995). Silencing the Past: Power and the Production of History Boston: Beacon.

Trouillot, M.R. (2007). El poder en el relato. Arqueología suramericana, 3 (2),162-183.

Vasco, G., Dagua, A. \& Aranda M. (1993). En el segundo día, la Gente Grande (Numisak) sembró la autoridad y las plantas y, con su jugo, bebió el sentido". En F. Correa. (Ed.), Encrucijadas de Colombia amerindia (pp. 9-48). Bogotá: Instituto Colombiano de Antropología.

Welters, L. (1992). Greek Folk Dress: Application of the Ethnohistorical Method. Clothing and Textiles Research Journal, 10 (3), 29-35. 